\title{
EFEITO DE ATRIBUTOS ASSOCIADOS AO TRABALHO SOBRE A EFICIÊNCIA DA INSENSIBILIZAÇÃO NO ABATE DE BOVINOS
}

\author{
Bianca Gonçalves da Costa ${ }^{1}$, Beatriz Temponi ${ }^{1}$, Claudia Pignatta de Macedo \\ Ferreira $^{1}$, Stavros Platon Tseimazides ${ }^{2}$, Augusto Hauber Gameiro ${ }^{1}$ \\ 1 FMVZ-USP \\ 2 Grupo Marfrig \\ Correspondência: Augusto Gameiro: gameiro@usp.br
}

\begin{abstract}
RESUMO: O Brasil é o maior exportador mundial de carne bovina e o segundo maior produtor de bovinos. Apesar desta posição, ainda se questionam diversos aspectos, dentre eles questões éticas como a realização de abate humanitário dos animais. Esta pesquisa teve como objetivo investigar o efeito de atributos associados ao trabalho humano sobre a eficiência do processo de insensibilização de bovinos. Foram levantados dados de um frigorífico de grande porte por meio do acompanhamento de um trabalhador no momento do disparo da pistola pneumática. Exames foram realizados nos animais atordoados. Um modelo de regressão logística e dois de regressão linear foram estimados sobre uma amostra de 2.150 animais. Observou-se que as variáveis que representam o raio de distância do tiro em relação ao local recomendado e a pressão da pistola de insensibilização apresentaram influência significativa sobre a probabilidade de o animal ser efetivamente insensibilizado. Já a hora sequencial do turno de trabalho da pessoa que realizava o atordoamento não apresentou efeito significativo sobre a probabilidade de insensibilização. $O$ número de disparos realizados em um mesmo animal não foi significativamente influenciado pela distância do primeiro tiro em relação ao alvo, nem pela hora sequencial de trabalho do insensibilizador. A hora sequencial do turno de trabalho do insensibilizador também não apresentou influência estatisticamente significativa sobre a distância do primeiro tiro realizado.
\end{abstract}

Palavras-chave: abate humanitário; bem-estar animal; pistola pneumática

\section{EFFECT OF LABOR ATTRIBUTES ON THE EFFICIENCY OF CATTLE SLAUGHTER STUNNING}

\begin{abstract}
Brazil is the largest beef exporter and second largest producer of cattle in the world. Despite this position there are several questions regarding some aspects, including ethical issues such as the realization of humane slaughter of animals. This research aimed to investigate the effect of labor attributes on the efficiency of cattle stunning. The data were collected from a large slaughterhouse through the monitoring of a worker at the time of firing the air gun. Examinations were performed in stunned animals. A logistic regression model and two linear regression ones were estimated on a sample of 2,150 animals. It was observed that the variables that represent the driving distance of the shot over the recommended location and pressure of the stunning gun have significant effect on the likelihood that the animal is effectively numb. The time of the sequential shift of the person who performed the stun had no significant effect on the likelihood of desensitization. The number of shots, performed in a same animal was not significantly influenced by the distance of first shot in relation to target, nor by hours of sequential work. The time sequential shift the insensibilizador also showed no statistically significant influence on the distance from the first shot done.
\end{abstract}

Key Words: animal welfare; humane slaughter; pneumatic gun 


\section{INTRODUÇÃO}

O Brasil é o maior exportador mundial de carne bovina e o segundo maior produtor de bovinos, com um rebanho de mais de 205 milhões de animais (IBGE, 2009; ABIEC, 2011). Apesar desta posição de destaque, ainda se questionam diversos aspectos relacionados ao sistema agroindustrial da pecuária bovina no país, especialmente questões sanitárias, ambientais e éticas. Neves (2008) afirma que a preocupação dos consumidores em relação ao bem-estar de animais de produção é crescente, principalmente na Europa, que a cada ano impõe novas leis relacionadas ao manejo do pré-abate, que engloba o transporte, insensibilização e abate de animais para consumo.

Roça (2002) considera que a insensibilização é a operação mais crítica de todo o processo e tem por objetivo colocar o animal em estado de inconsciência durante toda a sangria, sem oferecer sofrimento desnecessário, obtendo a sangria mais efetiva possível.

O método mecânico percussivo penetrante utiliza a pistola com dardo cativo e em bovinos é o método eleito para a insensibilização (Roça, 2002; MAPA, 2000). A pistola deve ser posicionada de modo a assegurar que 0 dardo penetre no córtex cerebral, através da região frontal do crânio. $O$ dardo cativo penetrante causa lacerações no tecido nervoso, porém não deve causar a morte. $\mathrm{O}$ animal deve morrer na sangria (STEPS, 2010). Além da correta pressão da pistola (mantida pela sua correta manutenção e limpeza), o funcionário que realiza esta operação é de vital importância para que a insensibilização seja adequada, pois deve acertar o ponto correto.

Para Grandin (2000) o processo de insensibilização pode ser prejudicado pelos seguintes agravantes: falta de manutenção dos equipamentos, falta de treinamento dos funcionários, cartuchos de munição úmidos e agitação dos animais.

Segundo Neves (2008) o cansaço dos funcionários, em conjunto com a falta de manutenção dos equipamentos, são fatores de risco para o erro do local de disparo. Após os treinamentos, os funcionários passam a aplicar 0 conhecimento no dia-a-dia, mas há a necessidade de treinamentos constantes, pois muitos funcionários, após longos períodos sem treinamento, podem voltar a manusear os animais de maneira errada (Grandin, 2006). Funcionários treinados para entender do comportamento dos animais também manejam 0 gado de forma mais tranquila (Grandin, 2006).

Para Gonzaga et al. (2003) "a produtividade marginal da hora trabalhada tende a ser uma função decrescente em horas médias, uma vez que após certo ponto os trabalhadores ficam menos produtivos". Essa produtividade marginal da hora média, para Estevão (1993), pode ser crescente para menores quantidades de horas trabalhadas devido a intervalos durante o trabalho, como almoço, lanches, etc.

Frost \& Andersen (1999) em estudo com funcionários de frigoríficos relatam que os movimentos repetitivos e que impõem pesos entre $5-10 \mathrm{~kg}$ (faixa de peso que se enquadra a pistola pneumática) sobre os ombros, eventualmente geram lesões musculoesqueléticas que podem surgir após 5 a 25 anos de trabalho.

Um funcionário cansado por muitas horas de trabalho pode ter diminuída sua capacidade cognitiva e motora, 0 que influenciaria no acerto do tiro para a insensibilização, resultando em maior quantidade de tiros e animais que sofrem o estresse e dor de receberem os tiros em locais inadequados. 
De acordo com Tseimazides et al. (2004) ao diminuir o estresse do gado ainda é facilitado o trabalho do operador da pistola, pois a reatividade animal é menor.

O processo de insensibilização eficaz consiste na perda imediata da consciência e pode ser verificado por meio de sinais que demonstrem a insensibilidade do animal à dor e aos estímulos do meio ambiente. Alguns testes são recomendados: testes à dor por pressão no septo nasal e língua; verificação de movimentos e reflexos oculares e palpebrais; contrações da mandíbula, região cervical e movimentos da coluna vertebral; pedaladas persistentes dos membros torácicos; movimentos de orelha e cauda; presença de respiração rítmica (Grandin, 1999; STEPS, 2010)

Entre os autores ainda há divergências sobre os métodos de avaliação da eficiência de insensibilização (Neves, 2008). Para Grandin (1999) a presença de respiração rítmica isolada já é um indicativo de sensibilidade. Porém Gregory et al. (2007) considera que a sua presença indica que não houve completa lesão medular, aumentando o risco de não ocorrer parada cardiorrespiratória e manutenção do fluxo sanguíneo ao cérebro, mas não indica sensibilidade isoladamente. Segundo o Programa Nacional de Abate Humanitário (STEPS), realizado pela Sociedade de Proteção Animal (WSPA), a presença da respiração rítmica, reflexos oculares, vocalização e movimentos persistentes de membros torácicos são indicativos de animal sensível. Pode ocorrer a respiração final chamada "gasp", caracterizada por profunda e única respiração e a extensão ou contração de membros torácicos no momento do corte da barbela e vasos sanguíneos (STEPS, 2010). Este momento muitas vezes é confundido com sensibilidade presente.
Esta pesquisa teve como objetivo investigar 0 efeito de atributos associados ao trabalho humano sobre a eficiência do processo de insensibilização de bovinos. Os atributos considerados foram: a distância do tiro ao ponto ideal recomendado, a pressão da pistola e a hora sequencial do turno de trabalho da pessoa que realizava 0 atordoamento.

Foram levantados dados de um frigorífico de grande porte por meio do acompanhamento do trabalho no momento do disparo da pistola pneumática e de exames realizados nos animais atordoados. No total, 2.150 bovinos foram pesquisados. Modelos estatísticos de regressão (logística e linear) foram estimados para se avaliar e quantificar o efeito do tempo de trabalho sobre a eficiência da insensibilização, bem como de outras variáveis relevantes, como a distância do disparo em relação ao ponto tecnicamente recomendado, a pressão da pistola e o número de disparos realizados.

\section{MATERIAL E MÉTODOS}

\section{Treinamento para coleta de dados}

Um treinamento para coleta de dados foi realizado no período de 19 a 30 de julho de 2010, em um frigorífico no interior do estado de São Paulo. O frigorífico possuía Sistema de Inspeção Federal e atendia ao mercado interno. Com a capacidade de abate entre 200 e 500 animais por dia, a jornada de trabalho no frigorífico ocorria no período da manhã, com início por volta das 5h30 e término ao final de todos os animais abatidos, variando conforme o número de animais e problemas técnicos enfrentados. A insensibilização era realizada através de pistola de dardo cativo com penetração. O objetivo desta pré-coleta foi o treino dos pesquisadores na anotação do número de disparos, local de disparo e verificação dos sinais 
de sensibilidade. Problemas técnicos interferentes no processo de insensibilização como a falta de manutenção da pistola, falta de contenção dos animais no box de atordoamento, risco ao pesquisador para verificar local exato do disparo e incapacidade do compressor de pistola de penetração inviabilizaram o uso dos resultados nas análises estatísticas. Ainda assim, o pré-experimento foi de suma importância para o treinamento dos pesquisadores e para o ajuste do método de coleta de dados para o experimento principal.

\section{Coleta dos Dados}

A coleta de dados foi realizada durante o mês de abril de 2011 em outro frigorífico, sendo este de grande porte, localizado no interior do estado de São Paulo. O frigorífico possuía Sistema de Inspeção Federal e atendia o mercado interno e externo. A jornada de trabalho durante o período das coletas ocorreu no período da manhã, com início às 6 horas e término ao final de todos os animais abatidos, variando conforme 0 número de animais e problemas técnicos enfrentados. Tal jornada compreendia ao redor de nove horas diárias, sendo uma hora para almoço.

A insensibilização era realizada com pistola de dardo cativo com penetração, recebendo manutenção diária. O tronco de contenção total possuía piso antiderrapante, parede móvel, pescoceira, sistema mecânico que conduzia 0 animal até 0 box (carrinho), painel de comando com o objetivo de reduzir o desgaste do operador, segundo especificação do fabricante. A pistola de atordoamento era da marca Jarvis (modelo USSS-1), com penetração e disparo somente quando entra em contato com a cabeça do animal; pressão de operação máxima entre 160 a 190 psi; peso de $14,7 \mathrm{~kg}$ com contrapesos que diminuem $\mathrm{o}$ esforço do funcionário para utilizá-la; diâmetro da haste de penetração de 15,9 mm (Jarvis, 2012).

A pesquisa foi realizada durante quatro dias. Um pesquisador ficou próximo ao funcionário que realizava a insensibilização coletando os dados do número de disparos e pressão da pistola. Outro pesquisador observava os sinais de sensibilidade na calha de sangria. $O$ terceiro pesquisador, também na calha de sangria, coletava os dados de local de disparo. Um mesmo trabalhador ficou responsável pela atividade de atordoamento durante todo o período de coleta de dados.

Os sinais de sensibilidade observados foram escolhidos com base no recomendado pelo STEPS (STEPS, 2010). Foram coletados os seguintes dados: i) reflexos oculares e palpebrais por pressão digital; ii) presença de respiração rítmica; iii) reação à dor por pressão da língua e do septo nasal; iv) rigidez de mandíbula; v) movimento de orelhas; e vi) vocalização. A respiração foi observada a partir da movimentação do flanco e por sensibilidade de ar expirado sentido no verso da mão do pesquisador. Os animais foram avaliados, no máximo, em até 60 segundos após o disparo, não havendo o risco de avaliar animais que poderiam possivelmente voltar à consciência de acordo com o recomendado pelo STEPS (2010).

O local de disparo foi analisado baseando-se no método proposto por Gallo et al. (2003) utilizando-se um alvo de material plástico transparente com diâmetro de $16 \mathrm{~cm}, 8$ círculos concêntricos equidistantes em $2 \mathrm{~cm}$ e círculo central com diâmetro de $2 \mathrm{~cm}$ tendo o centro apontado como alvo (Figura 1). O local do disparo era classificado em até 2, 4, 6 ou $8 \mathrm{~cm}$ do alvo.

Durante o período de coleta a pistola apresentou ligeira instabilidade de pressão em alguns momentos, 
funcionando com pressão mínima de até 115 psi. Os dados foram utilizados para correlacionar a pressão com a eficiência de insensibilização.

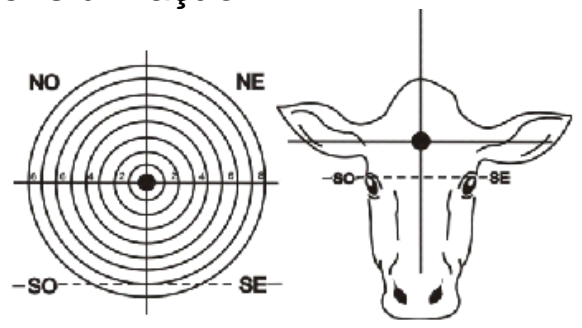

Figura 1 - Representação do alvo utilizado e seu posicionamento no crânio do animal para identificar o local do disparo e sua distância do alvo central

\section{Modelos estatísticos de regressão}

Quando a variável dependente apresenta natureza dicotômica os modelos de regressão linear não são adequados para a elaboração de modelos matemáticos, mas sim aqueles conhecidos como "logit" e "probit" (Allison, 1999).

O modelo de regressão logística, conhecido como "logit", apresenta a característica de limitar o valor da variável dependente entre 0 e 1 , independentemente das características e dos valores assumidos pelas variáveis explicativas. Portanto, para avaliar se um determinado animal foi ou não insensibilizado, o modelo logit pode ser utilizado. Tal modelo pode ser representado como se segue:

$$
\log \left[\frac{p_{i}}{1-p_{i}}\right]=\beta_{0}+\beta_{i} X_{i}
$$

Onde $p_{i}$ é a probabilidade de $y_{i}=$ 1. Fazendo a transformação para isolar $p_{i}$ tem-se:

$$
\begin{aligned}
p_{i} & =\frac{\exp \left(\beta_{0}+\beta_{i} X_{i}\right)}{1+\exp \left(\beta_{0}+\beta_{i} X_{i}\right)}, \mathrm{ou} \\
p_{i} & =\frac{1}{1+\exp \left(-\beta_{0}-\beta_{i} X_{i}\right)}
\end{aligned}
$$

Uma propriedade importante dessa expressão é que, para qualquer valores para $\beta$ e $X$, o valor de $p_{i}$ estará entre 0 e 1 . Assim, ao serem obtidos os parâmetros do modelo $(\beta)$ por meio da regressão logística, pode-se estimar a probabilidade de ocorrência do evento em análise por meio de valores assumidos pelas variáveis explicativas $(X)$. Uma apresentação detalhada do modelo logit pode ser obtida em So (1995).

Além do modelo logit, duas regressões lineares foram realizadas. Assim, para a análise dos dados do experimento foram estimados três modelos de regressão, que são descritos na sequência.

\section{Modelo 1}

No modelo 1 a variável binária dependente era 0 estado de insensibilização ou não do animal (sens). Adotou-se o valor 0 para 0 animal insensibilizado e 1 para o animal sensível. As variáveis explicativas foram: i) a distância do tiro ao ponto ideal recomendado (dist), podendo assumir os valores 2, 4, 6 ou 8, em centímetros; ii) a pressão da pistola (pres), em psi; e iii) a hora sequencial do turno de trabalho da pessoa que realizava o atordoamento (hour) no momento do disparo, assumindo valores de $1,2,3,4,5,6,7$ ou 8. Para processar o modelo foram utilizadas aquelas observações caracterizadas por apenas um disparo. Utilizou-se 0 modelo logit binário:

$$
\text { sens }_{i}=\frac{1}{1+\exp \left(-\beta_{0}-\beta_{1} \text { dist }_{i}-\beta_{2} \text { pres }_{i}-\beta_{3} \text { hour }_{i}\right)}
$$

Sendo sens $s_{i}$ a probabilidade de o animal $i$ continuar sensível após 0 processo de insensibilização. Dessa forma, o modelo 1 foi utilizado para testar a hipótese de que a distância do (primeiro) tiro em relação ao alvo recomendado, a pressão da pistola e a hora sequencial de trabalho do insensibilizador interferem na probabilidade de 0 animal não ser adequadamente insensibilizado no processo. O modelo 1 foi processado utilizando-se a função "PROC LOG", do software SAS 9.2., por meio do método de máxima verossimilhança. 


\section{Modelo 2}

No modelo 2 a variável dependente era o número de disparos em um mesmo animal (ndis). As variáveis explicativas foram as mesmas do modelo 1. Para processar o modelo foram utilizadas todas as observações obtidas, e não apenas aquelas caracterizadas por apenas um disparo. Utilizou-se o modelo de regressão linear múltipla:

ndis $_{i}=\beta_{0}+\beta_{1}$ dist $_{i}+\beta_{2}$ pres $_{i}+\beta_{3}$ hour $_{i}$

Por meio da utilização deste segundo modelo, procurou-se testar a hipótese de que a distância do primeiro tiro em relação ao alvo recomendado, a pressão da pistola e a hora sequencial de trabalho do insensibilizador interferem no número de disparos realizados.

No modelo 1 aquelas observações caracterizadas por mais de um disparo não foram consideradas, uma vez que disparos adicionais certamente influenciam no sucesso de insensibilização dos animais. Portanto, o modelo 2 foi proposto como complementar ao primeiro.

O modelo 2 foi processado utilizando-se a função "PROC REG", do software SAS 9.2., por meio do método mínimos quadrados ordinários.

\section{Modelo 3}

No terceiro modelo, a variável dependente era a distância do primeiro tiro em relação ao alvo recomendado (dist); e a variável explicativa a hora sequencial do turno de trabalho da pessoa que realizava $o$ atordoamento (hour) no momento do disparo, assumindo valores de 1, 2, 3, 4, 5, 6, 7 ou 8. Utilizou-se o modelo de regressão linear simples:

$$
\text { dist }_{i}=\beta_{0}+\beta_{1} \text { hour }_{i}
$$

Pela aplicação deste modelo procurou-se testar a hipótese de que a hora de trabalho apresenta influência significativa na distância do tiro, ou, em outras palavras, sobre a precisão do tiro. Assim como no modelo 2, foram utilizadas todas as observações obtidas, e não apenas aquelas caracterizadas por apenas um disparo. O modelo 3 foi processado utilizando-se a função "PROC REG", do software SAS 9.2., por meio do método mínimos quadrados ordinários.

\section{RESULTADOS E DISCUSSÃO}

Para o processamento do modelo 1 foram utilizadas 2.037 observações, sendo cada uma representada por um animal. Nessa amostra, 98 observações disseram respeito a animais sensíveis (sens $=1$ ) e 1.939 a animais insensíveis $($ sens $=0)$, segundo critérios analíticos previamente descritos. Os testes de hipótese de nulidade global dos parâmetros do modelo (razão de verossimilhança, escore e Wald), representados pelas respectivas estatísticas de qui-quadrado, apresentaram valores de $\mathrm{P}$ menores do que 0,050 , permitindo a rejeição da hipótese de que todos os parâmetros são iguais a zero. Portanto, pelo menos uma das variáveis explicativas consideradas (dist, pres ou hour) apresentou influência significativa sobre a probabilidade dos animais continuarem sensíveis após 0 procedimento de insensibilização.

Tabela 1 - Análise das estimativas de máximaverossimilhança para o modelo.

\begin{tabular}{|c|c|c|c|c|c|}
\hline Efeito & Estimativa & $\begin{array}{l}\text { Desvio } \\
\text { padrão }\end{array}$ & $\begin{array}{c}\text { Qui- } \\
\text { quadrado }\end{array}$ & Valor $\mathrm{P}$ & $\begin{array}{c}\text { Razão } \\
\text { de } \\
\text { chances }\end{array}$ \\
\hline Intercepto & 0,5702 & 1,8499 & 0,0950 & 0,7579 & - \\
\hline dist' $^{\prime}$ & 0,1854 & 0,0894 & 4,2975 & 0,0382 & 1,204 \\
\hline $\begin{array}{l}\text { pres }^{2} \\
\text { hour }^{3}\end{array}$ & $\begin{array}{l}-0,0229 \\
-0,0194\end{array}$ & $\begin{array}{l}0,0108 \\
0,0516\end{array}$ & $\begin{array}{l}4,4837 \\
0,1411\end{array}$ & $\begin{array}{l}0,0342 \\
0,7072\end{array}$ & $\begin{array}{l}0,977 \\
0,981\end{array}$ \\
\hline
\end{tabular}

Observa-se que as variáveis que representam o raio de distância do tiro em relação ao local recomendado (dist) e a pressão da pistola de insensibilização (pres) apresentaram influência significativa sobre a probabilidade de 0 animal ser efetivamente insensibilizado. Já a hora sequencial do turno de trabalho da 
pessoa que realizava o atordoamento (hour), não apresentou efeito estatisticamente significativo sobre a probabilidade de insensibilização. Esse resultado pode ter sido alcançado pela experiência do funcionário, de mais de 20 anos atuando nesta função, o treinamento recebido, a adequada infraestrutura e os intervalos para descanso.

Ressalta-se que os funcionários tinham à sua disposição banheiro e bebedouros dentro do frigorífico, respeitando as normas de higiene preconizadas. Os intervalos para ir ao banheiro e área de fumo duravam cinco minutos, ocorrendo a cada duas horas ou duas horas e meia de trabalho. Era fornecido café da manhã e almoço aos funcionários. Existia área de descanso disponível. O frigorífico possuía medidas de controle de risco contra acidentes e níveis de ruído. Possuía, ainda, políticas internas de treinamentos e conscientização dos funcionários em relação ao bem-estar animal.

O resultado encontrado neste experimento diverge do proposto por Gonzaga et al. (2003), Estevão (1993) e Neves (2008) que consideram que o número de horas de trabalho do funcionário está relacionado com a eficiência do seu trabalho. Outros fatores também colaboram para que o tiro não ocorra adequadamente, como a reatividade dos animais. Segundo Tseimazides et al. (2004) animais muito reativos dificultam 0 acerto do tiro, levando ao maior cansaço dos funcionários, sofrimento dos animais e erros nos disparos.

A análise da razão de chances associada à variável dist mostra que a cada centímetro que o tiro distancia-se do alvo recomendado, aumenta em $20,4 \%$ a chance de o animal não ser efetivamente insensibilizado. No caso da pressão da pistola (pres) a cada psi de aumento na regulagem, reduz em $2,3 \%$ a chance de um animal permanecer sensível. Esse resultado é corroborado por Neves (2008) e Grandin (2000) que consideram essencial a boa manutenção dos equipamentos.

Fries et al. (2011) analisaram, em dois frigoríficos, 8.879 crânios de bovinos insensibilizados com pistola e encontraram $64,7 \%$ e $65,3 \%$ dos crânios que receberam 0 tiro corretamente e $4,0 \%$ e $3,1 \%$ que receberam o tiro com baixa precisão.

As Figuras 2 e 3 ilustram 0 resultado de simulações utilizando o modelo 1.

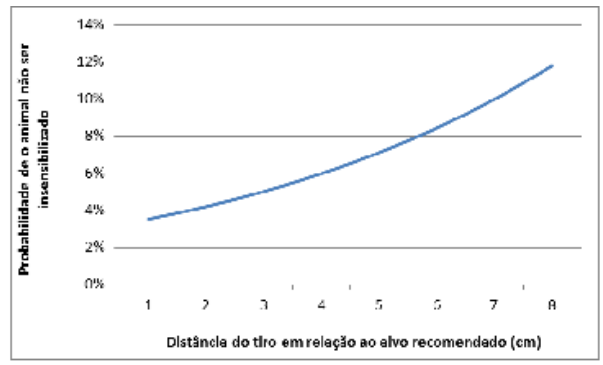

Figura 2 - Curva de probabilidade estimada de 0 animal não ser insensibilizado em função da distância do tiro em relação ao alvo recomendado (considerando pressão da pistola ajustada para 175 psi; e a terceira hora de turno de trabalho do insensibilizador).

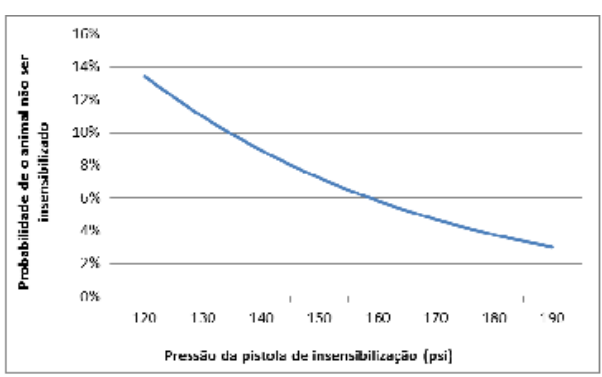

Figura 3 - Curva de probabilidade estimada de 0 animal não ser insensibilizado em função da pressão da pistola de insensibilização (considerando distância de $2 \mathrm{~cm}$ do tiro em relação ao alvo recomendado e; e a terceira hora de
turno de trabalho do insensibilizador).

Para o processamento do modelo 2 foram utilizadas 2.149 observações. A análise de variância obteve estatística $F$ igual a 32,53, com valor $P$ inferior a 0,0001 , permitindo a rejeição da hipótese de nulidade de todos os parâmetros associados às variáveis explicativas. A Tabela 2 apresenta 0 resultado das estimativas dos parâmetros do modelo.

Os resultados mostram que 0 número de disparos, realizados em um mesmo animal por ocasião da insensibilização, não é significativamente influenciado pela 
distância do primeiro tiro em relação ao alvo, nem pela hora sequencial de trabalho do insensibilizador. A pressão da pistola, contudo, apresentou influência significativa, tendo 0 parâmetro estimado, o sinal negativo. Este resultado é confirmado por Neves (2008) que obteve em seus resultados que a distância do primeiro disparo em relação ao alvo não influenciou o número de disparos recebidos.

\begin{tabular}{|c|c|c|c|c|}
\hline Efeito & Estimativa & $\begin{array}{l}\text { Desvio } \\
\text { padrão }\end{array}$ & Valor $\mathrm{t}$ & Valor $\mathrm{P}$ \\
\hline $\begin{array}{l}\text { Intercepto } \\
\text { disp' } \\
\text { hour } \\
\text { pres }^{3}\end{array}$ & $\begin{array}{l}2,17749 \\
-0,00643 \\
-0,00069 \\
-0,00634\end{array}$ & $\begin{array}{l}0,11646 \\
0,00489 \\
0,00251 \\
0,00068\end{array}$ & $\begin{array}{l}18,70 \\
-1,32 \\
-0,27 \\
-9,33 \\
\end{array}$ & $\begin{array}{l}<0,0001 \\
0,1882 \\
0,7836 \\
<0,0001\end{array}$ \\
\hline
\end{tabular}

Tseimazides et al. (2004) pesquisou a reatividade de bovinos no box de insensibilização e concluiu que animais cruzados são mais reativos, também confirmou que esses animais receberam maior número de disparos.

A Figura 4 traz o resultado de simulação utilizando o modelo 2 obtido. Por se tratar de um modelo de regressão linear, a curva obtida apresenta forma de reta, diferentemente da curva do modelo 1 (tipo logit).

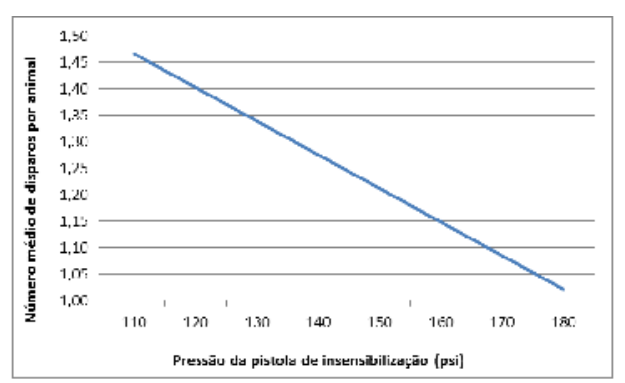

Figura 4 - Curva estimada do número médio de di sparos por animal em função da pressão da pistola de insensibilização (considerando distância de $2 \mathrm{~cm}$ do tiro em relação ao alvo recomendado ; e a terceira hora de turno de trabalho do insensibilizador).

Estando a pistola calibrada para uma pressão de 180 psi, o número médio de disparos aproxima-se de 1 (um), que pode ser considerado o ideal, pois demanda menos tempo para 0 processo, evitando mais estresse do animal e do funcionário. Por outro lado, com uma calibração de 110 psi, por exemplo, em média o insensibilizador executaria pouco mais de 1,45 tiros por animal, ou seja, para cada dois animais que passam pela insensibilização, seriam necessários praticamente 3 disparos. A pressão mínima recomendada pelo Programa Nacional de Abate Humanitário (STEPS) é de 160 psi para pistolas de dardo cativo com penetração Jarvis (modelo USSS-1). Deve-se sempre trabalhar de acordo com as especificações do fabricante da pistola, que no caso informa a pressão de operação sendo entre 160 e 190 psi (JARVIS, 2012). A fraca pressão da pistola não permite que os danos necessários ao sistema nervoso central ocorram, sendo necessário repetir os disparos.

Esse resultado reforça a importância da manutenção da pressão da pistola e aponta para o risco de penalidades em auditorias de bem-estar animal, nas quais é exigido que, no mínimo, 95\% dos animais sejam insensibilizados no primeiro tiro (Grandin, 2001). Andrade et al. (2008) avaliou a quantidade de disparos em comparação com a idade dos animais e relacionou o dia em que houve maior número de disparos por animal com a falta de limpeza da pistola. Segundo o autor a limpeza diária da pistola é necessária para evitar a existência de um zinabre no êmbolo da pistola, que prejudica 0 movimento do dardo, diminuindo sua força de impacto. A Instrução Normativa $n^{\circ} 03$ do MAPA, recomenda que os animais devam ser insensibilizados no primeiro disparo (MAPA, 2000). A limpeza e manutenção da pistola devem ser diárias sendo essenciais para seu correto funcionamento (STEPS, 2010).

O American Meat Institute considera aceitável o mínimo de 95\% dos animais insensibilizados no primeiro disparo. Pesquisa realizada em 10 frigoríficos americanos concluiu que $40 \%$ das plantas frigoríficas não 
obtiveram resultados aceitáveis por falta de manutenção adequada dos equipamentos (Grandin, 2006).

Para o processamento do modelo 3 também foram utilizadas as mesmas 2.149 observações do anterior. A análise de variância obteve estatística $F$ igual a 1,35 , com valor $P$ igual a 0,2447 , o que não permite a rejeição da hipótese de nulidade do parâmetro associado à variável explicativa, no caso a hora sequencial do turno de trabalho do insensibilizador. A Tabela 3 apresenta o resultado das estimativas dos parâmetros do modelo 3 .

Tabela 3 - Parâmetros estimados para 0 modelo 3 e suas respectivas \begin{tabular}{rcccc}
\hline Efeito & Estimativa & $\begin{array}{c}\text { Desvio } \\
\text { padrão }\end{array}$ & Valor t & Valor $\mathrm{P}$ \\
\hline Intercepto & 2,64472 & 0,04487 & 58,94 & $<0,0001$ \\
hour $^{r}$ & $-0,01248$ & 0,01073 & $-1,16$ & 0,2447 \\
\hline Hor a sequencial do turno de trabalho & & &
\end{tabular}

Assim, a hora sequencial do turno de trabalho do insensibilizador não apresentou influência estatisticamente significativa sobre a distância do primeiro tiro realizado por ele. Gregory et al. (2007) considera que fatores como falta de manutenção da pistola e treinamento dos funcionários interferem na eficiência do local de disparo. Para Grandin (2006), uma boa instalação não garante o bem-estar dos animais se os funcionários não realizarem os procedimentos de maneira adequada e cuidadosa. Segundo a autora, a gestão dos funcionários é essencial para que a manutenção, manuseio dos equipamentos e manejo dos animais sejam adequados. Embora as horas de trabalho não tenham influenciado, o fator mais importante foi a manutenção da pistola. A hora de trabalho pode não ter sido correlacionada com maior número de erros devido, provavelmente, à experiência do insensibilizador de 20 anos na mesma função, treinamentos e conscientização da importância do local adequado de disparo, boas instalações para trabalhar e períodos de descanso satisfatórios.

Estes fatos reforçam a idéia de que treinamentos constantes e boas condições de trabalho nos frigoríficos são essenciais para o bem-estar animal e bem-estar humano.

\section{CONCLUSÃO}

A hora sequencial de trabalho do insensibilizador não apresentou influência: i) sobre a probabilidade de sucesso na insensibilização; ii) sobre o número de disparos realizados; e iii) sobre a distância do tiro em relação ao alvo tecnicamente recomendado. A pressão da pistola foi a variável que melhor explicou a probabilidade de sucesso na insensibilização dos animais. Ademais, quanto maior a pressão reduz-se a necessidade de o insensibilizador ter que realizar mais de um disparo. Apesar de intuitivas essas conclusões, as mesmas servem para reiterar a importância do adequado manejo da pistola de insensibilização. A distância do tiro em relação ao alvo tecnicamente recomendado também apresentou influência significativa sobre o sucesso da insensibilização.

\section{AGRADECIMENTOS}

Os autores agradecem à Charli Beatriz Ludtke, Angelica Simone Cravo Pereira e Letícia Zoppa pelas importantes contribuições a esta pesquisa.

\section{REFERÊNCIAS}

ALLISON, P.D. Logistic regression using the SAS system: theory and application. Cary: SAS Publishing, 1999. 288 p.

ANDRADE, E.N.; ROÇA, R.O.; SILVA, R.A.M.S.; GONÇALVES, H.C.; PINHEIRO, R.S.B. . et al. Insensibilização de bovinos abatidos no Pantanal Sul-Mato-Grossense e ocorrência de lesões em carcaças. Ciência Animal Brasileira, v.9, n.4, p.958-968, 2008.

ASSOCIAÇÃO BRASILEIRA DAS INDÚSTRIAS EXPORTADORAS DE CARNES - ABIEC.

Produção Mundial de Carne, 2011. Disponível 
em<http://www.abiec.com.br/download/stat_mer cadomundial.pdf>. Acesso em: 10/12/2011.

BARBALHO, P.C.; LUDTKE, C. Abate humanitário de bovinos. Workshop de Bioética e Bem-Estar aplicados aos Animais de Produção. Nova Odessa: Instituto de Zootecnia, 2009.

ESTEVÃO, M. Employment level, hours of work, and labor adjustment cost in the Brazilian industry. Revista Brasileira de Economia, v.47, n.2, p.205-42, 1993.

FRIES R.; SCHOHE, K.; LOTZ, F.; ARNDT, G. . et al. Application of captive bolt to cattle stunning - a survey of stunner placement under practical conditions. Animal, Available on CJO doi:10.1017/S1751731111002667. Acesso em $24 / 01 / 2012$

FROST, P.; ANDERSEN, J.H. Shoulder impingement syndrome in relation to shoulder intensive work. Journal of Occupational and Environmental Medicine, v.56, n.7, p.494-498, 1999.

GALLO, C.; TEUBER, C.; CARTES, M. et al. Mejoras em la insensibilización de bovinos com pistola neumática de proyectil retenido tras câmbios de equipamento y capacitación del personal. Archivos de Medicina Veterinaria, v.35, n.2, p.159-170, 2003.

GONZAGA, G.M.; MENEZES FILHO, N.A.; CAMARGO, J.M. Os efeitos da redução da jornada de trabalho de 48 para 44 horas semanais em 1988. Revista Brasileira de Economia, v.57, n.2, p.369-400, 2003.

GRANDIN,T. Recommended ritual slaughter practices to improve animal welfare and employee safety. 1999. Disponível em < http://www.grandin.com/ritual/ritual.slaughter.tips .html >. Acesso em 03/07/2011.

GRANDIN, T. Return to sensibility problems after penetrating captive bolt stunning of cattle in commercial slaughter plants. Journal of the American Veterinary Medical Association, v.221, p.1258-1261, 2000.

\section{GRANDIN, T. Cattle Slaughter Audit Form} Based on American Meat Institute Guidelines, 2001. Disponível em < http://www.grandin.com/cattle.audit.form.html >. Acesso em 03/02/2011.
GRANDIN, T. Progress and challenges in animal handling and slaughter in the U.S. Applied Animal Behaviour Science, v.100, n.1, p.129139, 2006.

GREGORY, N.; LEE, C.J.; WIDDICOMBE, J.P. Depth of concussion in cattle shot by penetrating captive bolt. Meat Science, v.77, n.4, p.499-503, 2007.

INSTITUTO BRASILEIRO DE GEOGRAFIA E ESTATÍSTICA - IBGE. Banco de Dados

Eletrônico; 2009. Disponível em:

$<$ http://www.ibge.gov.br>. Acesso em 15/02/2010.

JARVIS. Manual de operação, manutenção e limpeza dos modelos USSS-1 e USSS-2. JARVIS ${ }^{\circledR}$ Products Corporation. 2012. Página: 16.

MINISTÉRIO DA AGRICULTURA, PECUÁRIA E ABASTECIMENTO - MAPA. Regulamento técnico de métodos de insensibilização para o abate humanitário de animais de açougue. Instrução Normativa n.3, de 17 de janeiro de 2000.

NEVES, J.E.G. Influências de Métodos de Abate no Bem-Estar e na Qualidade da Carne de Bovinos, 2008. Disponível em < http://www.fcav.unesp.br/download/pgtrabs/zoo/ m/3486.pdf >. Acesso em 12/12/2010.

ROÇA, R.O. Abate humanitário de bovinos, I Conferência virtual global sobre produção orgânica de bovinos de corte, 2002, p.6

SO, Y. A tutorial on logistic regression. Cary: SAS Institute Inc., 1995. Disponível em: < http://www.ats.ucla.edu/stat/sas/library/logistic.p df >. Acesso em: 08/08/2011.

\section{STEPS. Programa Nacional de Abate}

Humanitário: Melhorando o bem-estar animal no abate. CD-RM. 2010.

TSEIMAZIDES, S.P.; BARBALHO, P.C.; PARANHOS DA COSTA, M.J.R. Avaliação da reatividade de bovinos no box de atordoamento. In: XXII ENCONTRO ANUAL DE ETOLOGIA, 22, 2004, Campo Grande. Anais... Campo Grande: Sociedade Brasileira de Etologia, 2004. CD-ROM 